Supplementary information for

\title{
Deglacial abrupt climate changes: not simply a freshwater problem
}

\author{
Jorge Alvarez-Solas $(1,2)$, Marisa Montoya $(1,2)$ and Alexander Robinson $(1,2)$ \\ 1) Universidad Complutense de Madrid,28040 Madrid, Spain. \\ 2) Instituto de Geociencias, CSIC-UCM,28040 Madrid, Spain
}

\section{Contents of this file}

Figures $\mathrm{S} 1$ to $\mathrm{S} 3$ and their associated explanatory text and figure captions. The text is divided into two sections:

\section{Global versus Northern Hemisphere sea-level reconstructions for freshwater forcing}

Figure $\mathrm{S} 1$ shows the evolution of the AMOC when the model is forced with FWFs derived from the GLAC-1C and ICE-6G global sea-level reconstructions and applied to the North Atlantic ocean. Dashed lines show the same information but considering only the Northern Hemisphere $(\mathrm{NH})$ contributions to the global sea level record. Note that the differences between the thick and the dashed lines (hence, the Antarctic contribution) start to be noticeable from ca. $15 \mathrm{ka} \mathrm{BP}$ but remain small until ca. $13 \mathrm{ka}$ BP. In other words, the Antarctic contribution to the deglacial sea-level rise is of a minor amplitude compared to the $\mathrm{NH}$ one until ca. $13 \mathrm{ka} \mathrm{BP}$ in these reconstructions. Therefore applying the global or the $\mathrm{NH}$-only derived FWF produces significant different AMOC evolutions only after the Bolling-Allerod. 

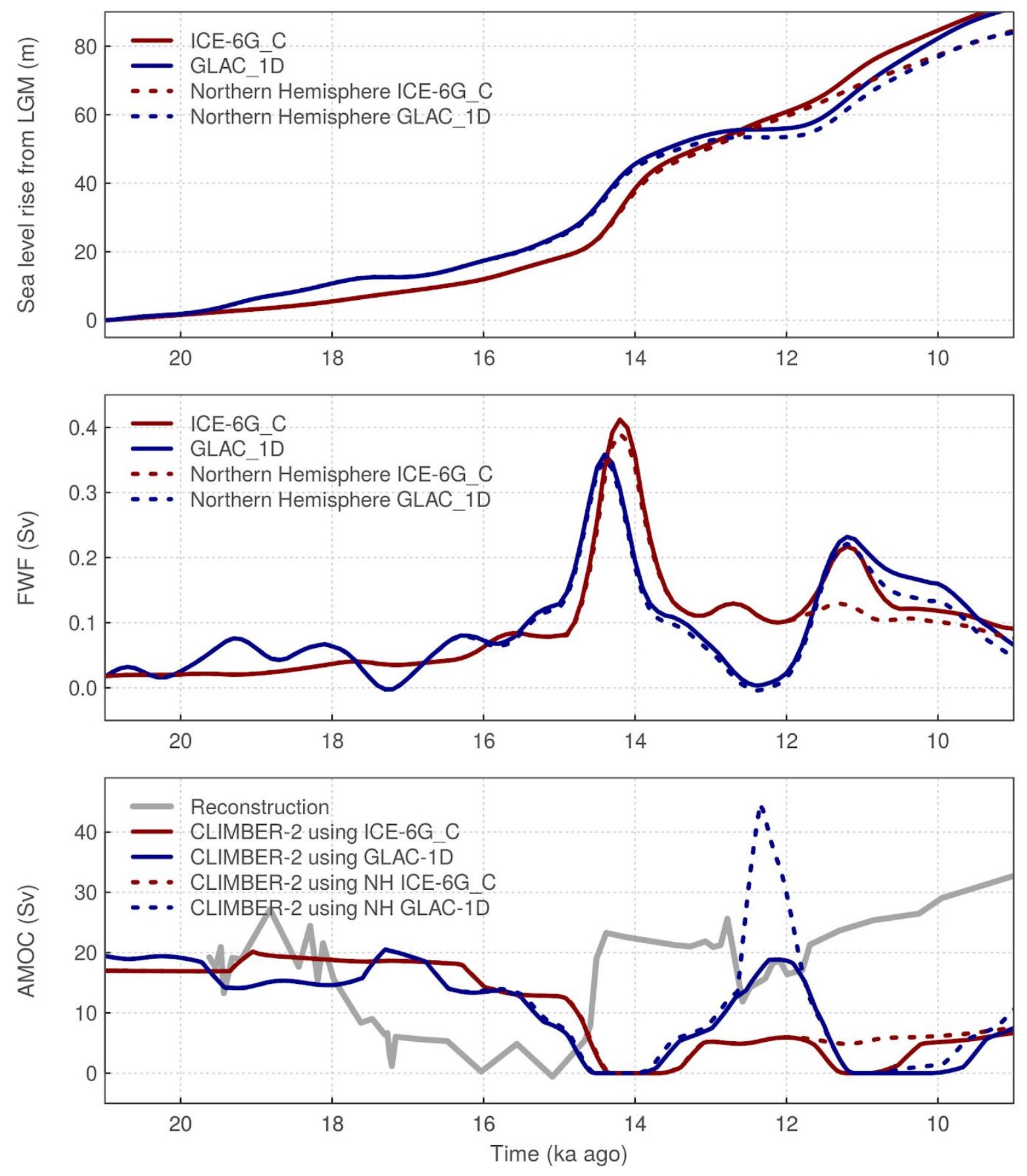

Figure S1 a) Reconstructed contributions to the ESL evolution (in meters from the LGM); global (thick lines) and Northern Hemisphere (dashed lines) according to Tarasov et al. [2012] (GLAC-1C; blue) and Peltier et al. [2015] (ICE-6G; red); b) FWF forcings (in Sv); d) simulated AMOC evolution. 


\section{Northern Hemisphere versus antarctic freshwater forcing}

We here address first the possibility of inducing an AMOC recovery as a result of a FWF around Antarctica. Figure S2 shows the simulated AMOC evolution when injecting water both in the North Atlantic and the Southern Ocean. Note that the NH FWF necessary to keep the AMOC on a halted state previous to the Bolling-Allerod is already inconsistent (of greater amplitude) with the global reconstruction. Figure S2 shows that an intermediate AMOC recovery can be promoted by means of antarctic FWF provided the circulation is already stopped. Nevertheless, it should be stressed that the antarctic freshwater quantities for this recovery to be of ca. $10 \mathrm{~Sv}$ are about 5 times greater than the global MWP-1a (red curve in Figure S2). Even for a slight recovery of ca. 3 Sv the Antarctic FWF needs to be of a greater amplitude than the global reconstructed meltwater injection during the MWP-1a. 

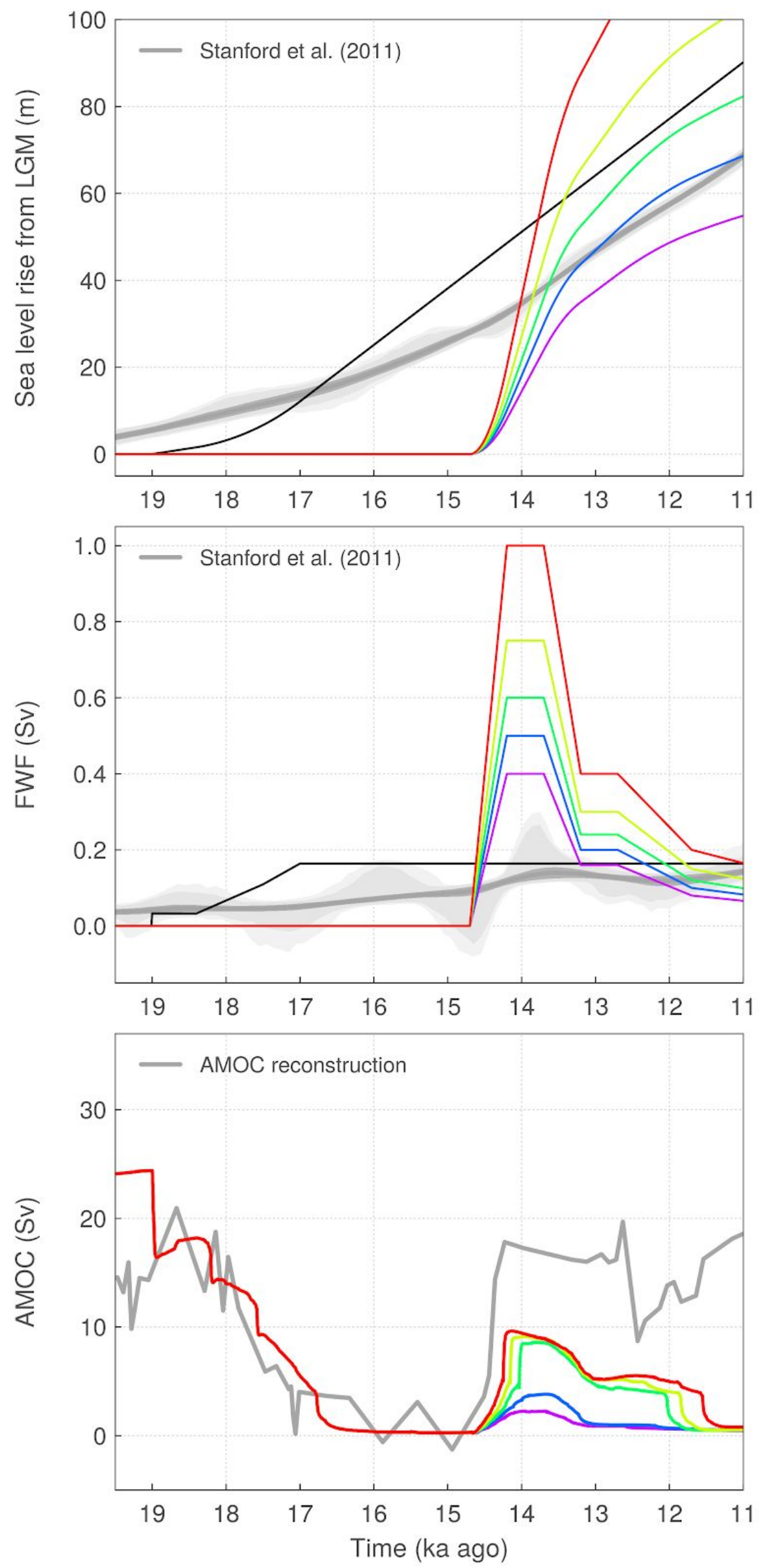
Figure S2 | a) Reconstructed ESL (in meters from the LGM) from Stanford et al. [2011] (grey palette) compared to the ESL used in this ensemble of simulations. The black line represents the Northern Hemisphere contribution while the color lines illustrate different Antarctic contributions to the sea-level increase; b) freshwater fluxes (FWF) in the North Atlantic (black) and different Antarctic contributions (color) (in Sv); c) simulated AMOC evolution for with CLIMBER-2 using each of the forcings FWFs.

Could a combination of $\mathrm{NH}$ and antarctic FWFs produce an AMOC evolution that is in good agreement with the $\mathrm{Pa} / \mathrm{Th}$ data and that at the same time is constrained to fulfill the global sea-level reconstruction? The ensemble of simulations shown in Figure S3 is conceived in order to answer this question. The $\mathrm{NH}$ and antarctic FWFs of a given simulation (represented by a given color) are constructed such that their addition gives the median reconstruction of Stanford et al, (2011), thus consistent with the global sea-level evolution. Additionally, as suggested by all the Northern Hemisphere ice-sheet reconstructions of the deglaciation, no removal of FWF (which would imply regrowth of the $\mathrm{NH}$ ice sheets) is allowed during the period previous to the Bolling-Allerod. Once these two requirements are imposed, the relative contribution of the $\mathrm{NH}$ and Antarctica are varied and the effects of this spread on the AMOC evolution is explored in the ensemble of Figure S3 (see main text for further interpretation). 

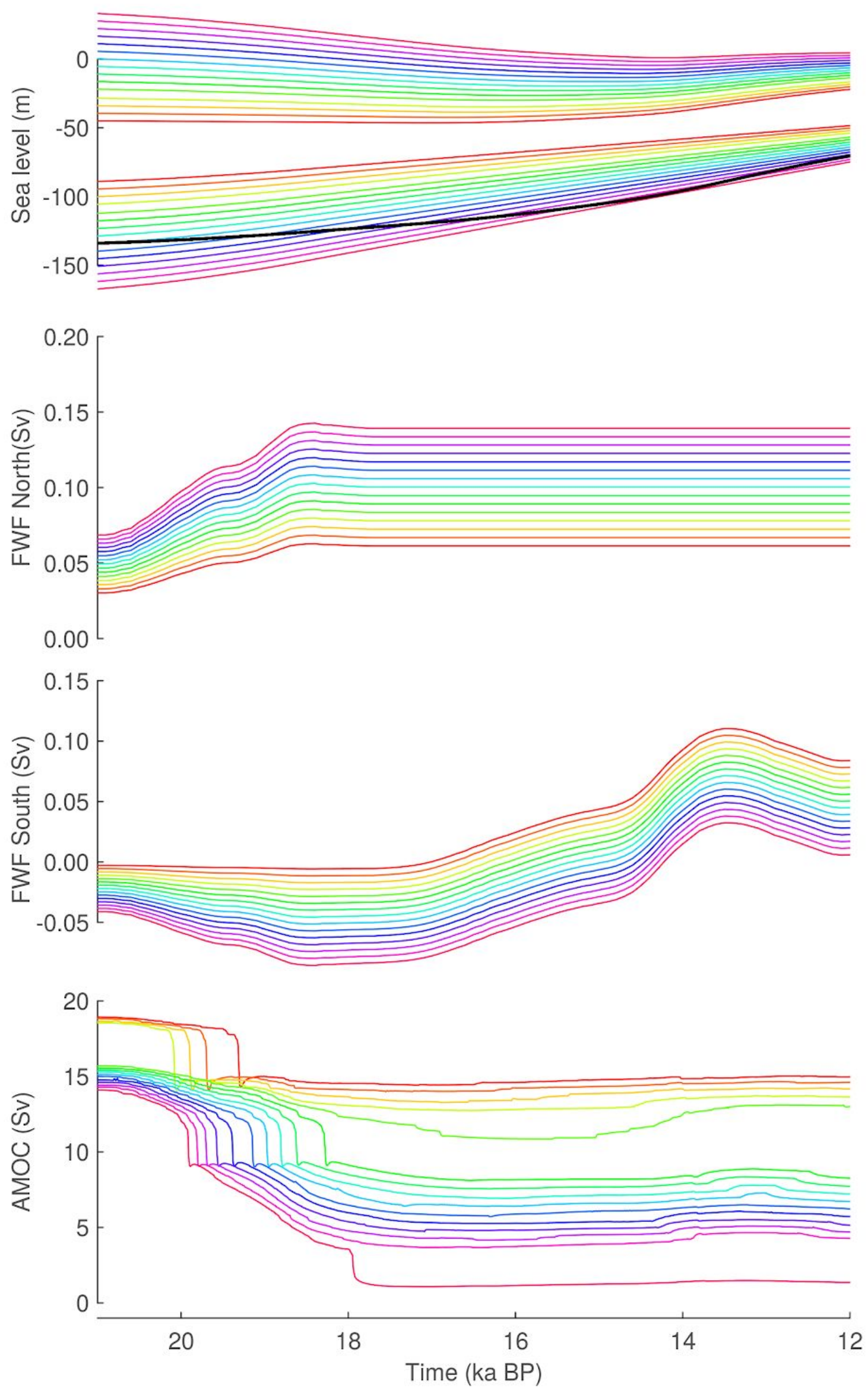
Figure S3 | a) Reconstructed contributions to the global ESL evolution from the $\mathrm{NH}$ and the $\mathrm{SH}$ (in meters; color lines). All the individual simulations (illustrated by a given color) are conceived such that the total sum $(\mathrm{NH}+\mathrm{SH})$ matches the median reconstruction of Stanford et al. [2011] (black curve). The upper part of panel a) shows the Antarctic contributions and the bottom part corresponds to the $\mathrm{NH}$ contributions to the global sealevel evolution (black curve); b) FWF in the North Atlantic and c) in the SH (in Sv); d) simulated $A M O C$ evolution. Note that the simulation represented by the lightest green shows a slight AMOC decrease from $19 \mathrm{ka} B P$ and a slight AMOC intensification during the Bølling Allerod. 